\title{
Detección de bocavirus humano en la población infantil de Tucumán y Santa Fe, Argentina
}

\author{
M. Verónica Vera-Garate, Juan Manuel Rudi, Alejandra Gómez, Fabiana Molina, \\ María Florencia Viotti, Lucila Ortellao, Judith Pierini, Natalia Sioli, Raquel Cociglio, Ana María Zamora, \\ Gustavo Ruiz de Huidobro, Dardo Costas, Susana López de Caillou, Stella Maris Sánchez, \\ Nora Mamaní, Rosanna Leva, Graciela Rodríguez, Sara Gallardo y Gabriela Kusznierz
}

\section{Detection of human bocavirus in children from Tucuman and Santa Fe, Argentina}

Background: A large proportion of acute respiratory tract infections (ARTI) remain without etiologic diagnosis, reason why new pathogens are investigated continuously. Human bocavirus (HBoV) was discovered in 2005, as a new member of Parvoviridae family and proposed to cause ARTI. Aim: To know the prevalence of HBoV among pediatric populations hospitalized for ARTI in two provinces of Argentina: Santa Fe and Tucuman; and to describe epidemiological and clinical aspects associated to its detection. Materials and Methods: We studied nasopharyngeal aspirates of patients younger than 5 years old that were hospitalized during 2013 due ARTI. HBoV DNA was assayed using PCR described by Allander et al. Traditional viruses were studied by immunofluorescence. Personal, clinical and epidemiological data were collected in a standardized form. Results: The HBoV was detected in 7\% of the samples and was prevalent in spring and summer and in children younger of 2 years old. Other respiratory viruses were detected in $22 \%$ of HBoV positive samples. Discussion: We detected HBoV in these two provinces of Argentina. Further studies should be performed to determine if it's a recent infection or prolonged viral shedding.

Key words: Human bocavirus, pediatrics, respiratory tract infection.

Palabras clave: Bocavirus, niños, infección respiratoria aguda.

\section{Introducción}

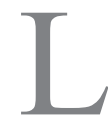

as infecciones respiratorias agudas (IRA) constituyen uno de los problemas de salud pública más importantes para la población infantil de 0 a 5 años y están dentro de las primeras cinco causas de mortalidad $^{1-3}$.

La etiología de la infección del tracto respiratorio es compleja y diversa, siendo los virus respiratorios los principales contribuyentes a la carga de la enfermedad respiratoria pediátrica ${ }^{4}$. Estudios previos han determinado que en un porcentaje significativo de infecciones respiratorias no se logra identificar el agente etiológico, lo cual sugiere que nuevos patógenos pueden estar involucrados ${ }^{5}$. Durante el año 2014, se estudiaron y notificaron 64.013 casos de infecciones respiratorias agudas en Argentina y sólo en 20.516 (32\%) se obtuvieron resultados positivos para algún virus respiratorio ${ }^{1}$.

Bocavirus humano (HBoV) fue descubierto en 2005 por Allander y cols., a partir de muestras respiratorias provenientes de niños ${ }^{6}$. Desde entonces se ha detectado en aspirados nasofaríngeos, suero y sangre de pacientes con infección respiratoria y en muestras de materia fecal de pacientes con IRA y/o gastroenteritis ${ }^{7}$.

Es un pequeño virus no envuelto, icosaédrico, perte- neciente a la familia Parvoviridae. Contiene un genoma de 5,3 kb de ADN cadena simple ${ }^{8}$ y no crece en líneas celulares estándares por lo cual su diagnóstico se ha basado en la detección del $\mathrm{ADN}^{9}$. El genoma de $\mathrm{HBoV}$ contiene tres marcos abiertos de lectura (en inglés open reading frames-ORFs). Los dos primeros codifican las proteínas no estructurales NS1 y NP1 y el tercero codifica las dos proteínas de la cápside viral: VP1 y VP2 ${ }^{9,10}$. Cuatro especies de HBoV han sido descubiertas: HBoV1 es la especie que predomina en el árbol respiratorio mientras que HBoV2, HBoV3 y HBoV4 se hallaron principalmente en muestras de materia fecal ${ }^{11}$. La secuencia de nucleótidos de las proteínas de la cápside VP1 y VP2 tiene una alta variabilidad, comparada con la de las proteínas no estructurales NS1 y NP1, por lo cual su análisis ha dividido a HBoV1 en dos genotipos: St1 y St2 ${ }^{12}$.

HBoV fue propuesto como causante de IRA, principalmente en lactantes ${ }^{6,4}$ y se lo detectó en tasas variables, desde 0,9 a $33 \%$, asociado a cuadro del tracto respiratorio superior, bronquiolitis, neumonía y exacerbación del asma $^{7,13-25}$. Sin embargo, HBoV tiene una elevada prevalencia en co-detecciones con otros patógenos ${ }^{7,10,13,14,19,20,22}$ y se detectó en muestras de individuos asintomáticos ${ }^{22,23,26}$ por lo cual aún debe ser confirmado su rol como patógeno.

Los objetivos de este estudio fueron investigar la
Santa Fe, Argentina.

Instituto Nacional de Enfermedades Respiratorias "Dr. Emilio Coni" (MVVG, JMR, AG, GK).

Hospital de Niños "Dr. Orlando Alassia" (FM, MFV, RC). Hospital "José Bernardo Iturraspe" (LO, JP, NS).

San Miguel de Tucumán, Argentina.

Hospital de Clínicas "Nicolás Avellaneda" (AMZ, G RdeH, DC, SLdeC, RL, GR, SG).

Hospital del Niño Jesús (SMS, NM)

Los autores declaran que no existen conflictos de intereses.

Fuente de financiamiento: no hubo

Recibido: 6 de octubre de 2015 Aceptado: 31 de diciembre de 2015

Correspondencia a: M. Verónica Vera Garate veritoverag@gmail.com 
prevalencia de $\mathrm{HBoV}$ en niños bajo 5 años de edad, hospitalizados por IRA, en dos provincias de Argentina: Santa Fe y Tucumán; y describir aspectos epidemiológicos y clínicos asociados a su hallazgo.

\section{Materiales y Métodos}

\section{Diseño y selección de participantes}

Estudio descriptivo, retrospectivo. Se incluyeron pacientes bajo 5 años de edad, con diagnóstico de IRA, internados durante el año 2013 en una de las siguientes instituciones: Hospital de Niños "Dr. Orlando Alassia" y Hospital "J. B. Iturraspe" de la ciudad de Santa Fe; y Hospital del Niño Jesús y Hospital de Clínicas "Nicolás Avellaneda" de la ciudad de Tucumán. Los cuatro hospitales son centros de mediana y alta complejidad y de referencia para las respectivas provincias.

Para la determinación del tamaño de muestra se consideró una prevalencia esperada de $21 \%$, con un nivel de confianza de $95 \%$ y una precisión de $4 \%$, con una población de 1.762 para la provincia de Santa Fe y de 1.253 para la provincia de Tucumán. El tamaño de muestra calculado fue de 325 y 302, respectivamente.

Para la determinación de co-detecciones con los virus respiratorios "clásicos" (definidos más adelante), se consideró un tamaño poblacional de 258 (pacientes cuya muestra fue positiva para virus clásicos) para Santa Fe y de 914 para Tucumán. El tamaño de muestra calculado fue de 157 y 277, respectivamente. La selección de las muestras fue aleatoria.

\section{Diagnóstico virológico}

Para la detección de $\mathrm{HBoV}$ se estudió un porcentaje de los aspirados nasofaríngeos (ANF) en los cuales no se identificaron virus respiratorios clásicos y un porcentaje de los ANF positivos. Los virus respiratorios clásicos que se estudiaron de rutina fueron: virus respiratorio sincicial (VRS), adenovirus (ADV), metapneumovirus (MPVh), parainfluenza e influenza A y B, mediante la técnica de inmunofluorescencia indirecta (IFI), utilizando anticuerpos monoclonales específicos e IgG de cabra anti $\mathrm{IgG}$ de ratón conjugado con isotiocianato de fluoresceína (Chemicon). La detección de influenza A y B se realizó, además, por reacción polimerasa en cadena en transcriptasa reversa (RPC-TR) en tiempo real ${ }^{27}$. Los ANF fueron conservados a $-70^{\circ} \mathrm{C}$ hasta la extracción del ADN, que se realizó a medida que se recibían las muestras de cada hospital, durante el año 2013 y 2014. La detección de $\mathrm{HBoV}$ se llevó a cabo en el Instituto Nacional de Enfermedades Respiratorias (INER) "Dr. Emilio Coni", mediante la RPC que amplifica la región N-terminal del gen de la proteína NP1, utilizando los siguientes cebadores: forward 5'-GAGCTCTGTAAGTACTATTAC $-3^{\prime}$ y reverse 5'-CTCTGTGTTGACTGAATACAG-3'6. La RPC se realizó en una mezcla de $25 \mathrm{ml}$ conteniendo: 2,5 ml de solución tampón DreamTaq Green Buffer 10X (Fermentas), la cual contiene $\mathrm{MgCl} 20 \mathrm{mM}$; 0,4 ml de cada uno de los cebadores (50 uM);0,4 ml de dNTPs (10 uM); 0,5 ml de DreamTaq polimerasa (Fermentas, $5 \mathrm{U} /$ $\mathrm{ml}) ; 5 \mathrm{ml}$ de ADN. El proceso de amplificación consistió en un paso inicial de desnaturalización a $94{ }^{\circ} \mathrm{C}$ durante 10 min, seguido de 35 ciclos: $94{ }^{\circ} \mathrm{C}$ por $1 \mathrm{~min}, 54{ }^{\circ} \mathrm{C}$ por $1 \mathrm{~min}$ y $72{ }^{\circ} \mathrm{C}$ por $2 \mathrm{~min}$. Los productos de RPC de $354 \mathrm{pb}$ se visualizaron mediante electroforesis en gel de agarosa al $2 \%$.

\section{Recolección de datos y análisis estadístico}

Se utilizó como instrumento una planilla clínicoepidemiológica estandarizada para recolectar datos personales, clínicos y epidemiológicos de los pacientes en los cuales se detectó ADN de bocavirus. Los datos se analizaron en el software EpiInfo ${ }^{\mathrm{TM}}$ 3.5.3.

Se definió enfermedad grave a los casos que requirieron internación en la Unidad de Cuidados Intensivos (UTI) o fallecieron.

Las variables continuas se resumieron como media o mediana (con su rango intercuartilo). Para variables categóricas, se calcularon los porcentajes. Los intervalos de confianza superior e inferior (IC) se estimaron para las proporciones, con un nivel de significación $(\alpha) 0,05$. Se utilizó el test $\chi^{2}$ Mantel-Haenszel para comparar variables discretas. Un valor de $\mathrm{p}<0,05$ fue considerado de significancia estadística. Se emplearon los programas EPIDAT 3.1 y SPSS IBM Statistics (versión 20).

\section{Resulltados}

\section{Frecuencia de detección de $\mathrm{HBoV}$}

Se procesaron 1.096 muestras respiratorias, de las cuales $46 \%$ (n: 504 ) procedieron de pacientes internados en hospitales de la ciudad de Santa Fe, y 54\% (n: 592) de la ciudad de Tucumán. Se detectó HBoV en 7\%; 95\% IC 6,23-7,7 (n: 78) del total de muestras analizadas. La positividad para la provincia de Santa Fe fue de 8,5\%; 95\% IC 7,28-9,72 (43) y para la provincia de Tucumán fue de 5,9\%; 95\% IC 4,91-6,89 (35).

\section{Estacionalidad}

HBoV fue detectado en todos los meses del año, excepto en noviembre. Los porcentajes de detección variaron mensualmente entre 1,4 y $63,6 \%$ para la provincia de Santa Fe; y entre 1,8 y 16,0\% para Tucumán (Figura 1). En las estaciones de primavera y verano se registró la mayor positividad.

En 28,2\% (n: 22) de los casos se observó co-detección con otros virus respiratorios. Los virus respiratorios clá- 


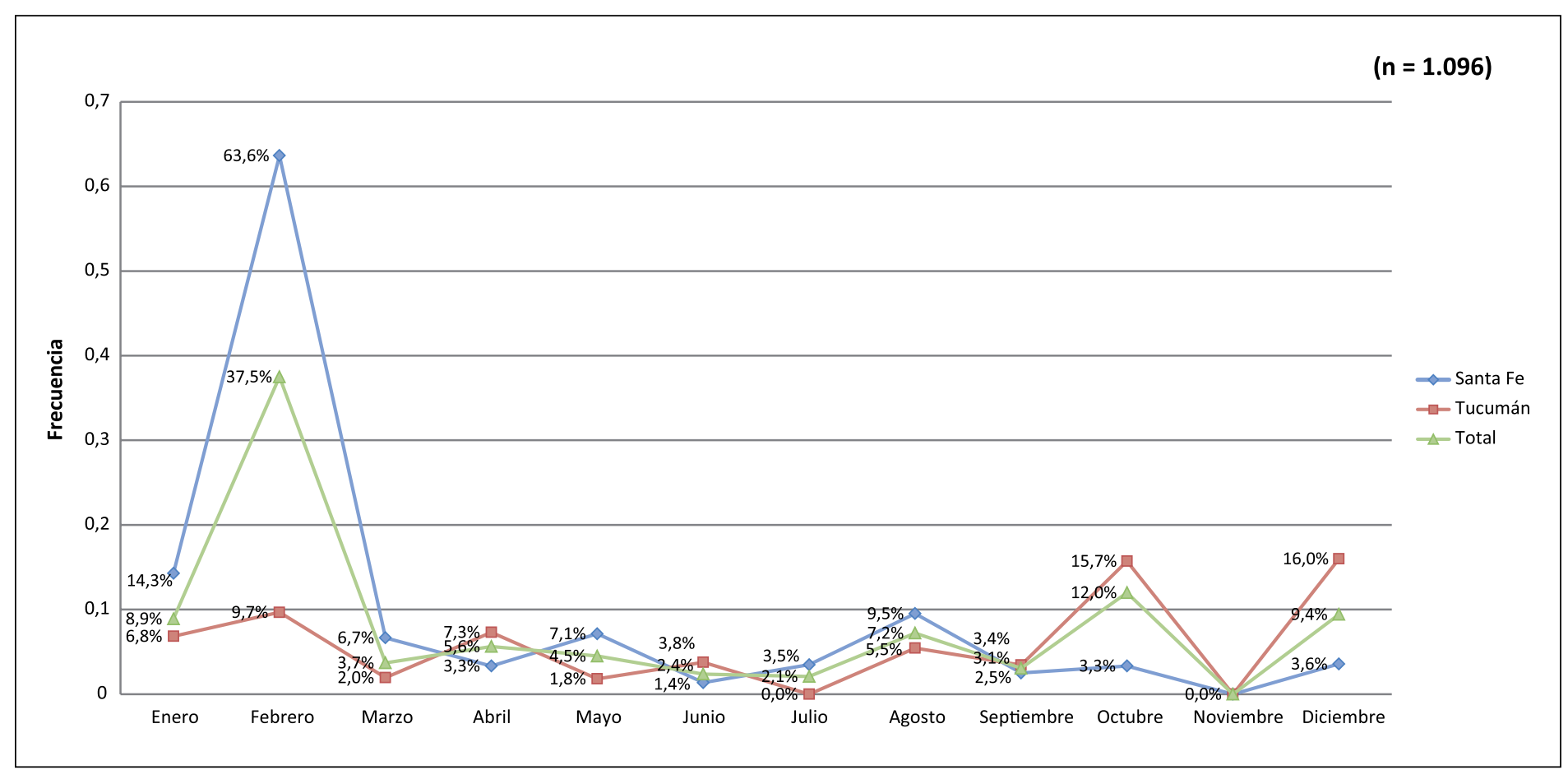

Figura 1. Distribución mensual de los casos positivos para HBoV durante el año 2013 para Tucumán, Santa Fe.

sicos hallados fueron VRS (n: 8); parainfluenza 3 (n: 6); MPVh (n: 3); ADV (n: 3) e influenza A (n: 2).

\section{Datos demográficos}

Los casos se distribuyeron en los siguientes grupos etarios: menor de 1 mes, 3 (4\%); 1-6 meses, 31 (40\%); 7-12 meses, 16 (21\%), 13-24 meses, 21 (27\%); 25-60 meses, $6(8 \%)$. La mediana de la edad correspondió a 8 meses (rango: 0,6 -50 meses). Cuarenta y dos pacientes (54\%) eran de sexo masculino. No se registraron diferencias estadísticamente significativas con respecto a la distribución por sexo, (p: 0,51).

\section{Manifestaciones clínicas}

Se recopilaron datos clínicos de 64 pacientes. El 53\% (n: 34) presentó internaciones previas debidas a enfermedad respiratoria y $41 \%$ (n: 26) antecedentes mórbidos (Tabla 1). Se encontró asociación entre la presencia de antecedentes mórbidos y el desarrollo de enfermedad grave (p: 0,047).

Los síntomas y signos se describen en la Tabla 1. La frecuencia respiratoria promedio de los pacientes fue de $51 / \mathrm{min}$, (rango de 20-80/min) y la saturación de oxígeno promedio de $94 \%$ (rango de $83-100 \%$ ).

Los diagnósticos de egreso más frecuentes fueron: neumonía, episodios de sibilancia y bronquiolitis (Tabla 1). Hubo dos casos fatales, ambos pacientes de dos meses de edad, uno de sexo masculino y otro femenino. Como antecedentes clínicos presentaban un síndrome genético (en estudio) y una cardiopatía congénita, respectivamente. El diagnostico de egreso fue neumonía para el varón y hemorragia pulmonar con shock séptico para la niña.

\section{Hallazgos radiológicos y de laboratorio}

El 78\% (n: 50) de los pacientes contaba con placa radiológica de tórax, de los que 10\% (n: 5) presentaba un patrón radiológico normal (Tabla 1). El 48\% (n: 31) de los casos, presentó leucocitosis, tres de éstos con linfocitosis (recuento de linfocitos $>9.000 / \mathrm{mm}^{3}$ ). La mediana del recuento de leucocitos fue de $11.600 / \mathrm{mm}^{3}$, (rango: $2.000-31.700 / \mathrm{mm}^{3}$ ). La mediana de la velocidad de sedimentación globular fue de $17 \mathrm{~mm} / \mathrm{h}$, con un rango de $3-99 \mathrm{~mm} / \mathrm{h}$.

\section{Medidas terapéuticas}

Requirió administración de oxígeno 77\% (n: 49) de los pacientes. Fueron internados en una unidad de cuidados intensivos $22 \%$ (n: 14), con una mediana de permanencia de 7 días (rango: 2-41 días). El 13\% (n: 8) de los pacientes requirió asistencia respiratoria mecánica. Con respecto a la estadía hospitalaria la mediana fue de 13 días (rango: 1-113 días). 
Tabla 1. Antecedentes clínicos, signos y síntomas, hallazgos radiológicos de tórax y diagnóstico de egreso

n $(\%)$

\section{Antecedentes clínicos}

Prematurez (< 37 semanas de gestación) 14 (22)

Cardiopatía congénita 8 (13)

Desnutrición $6 \quad$ (9)

Síndrome de Down 2 (3)

Inmunodeficiencia $1(1,6)$

Displasia broncopulmonar

$1(1,6)$

Malformaciones congénitas

$1(1,6)$

\section{Signos y síntomas}

Tos

Disnea

Fiebre

Rales subcrepitantes

Tiraje

Murmullo vestibular disminuido

Rinorrea

Sibilancias

Rechazo del alimento

Cianosis

Rales crepitantes

Distención abdominal

\section{Hallazgos radiológicos de tórax}

Rx normal

Atrapamiento aéreo

Patrón alveolar

Patrón intersticial

Atelectasia

Adenomegalias

\section{Diagnóstico de egreso}

Neumonía

Episodio de sibilancia

Bronquiolitis

Bronquitis

Cuadro de vías altas

Síndrome coqueluchoide

Síndrome bronquial obstructivo

Enfermedad tipo influenza

Broncoespasmo

Broncodisplacia pulmonar

\section{Impacto de la co-infección}

No se encontró asociación entre co-infección y la admisión en UTI ni con la duración de la estadía ( $\mathrm{p}$ : 0,80 y p: 0,26 , respectivamente). Además los pacientes co-infectados no tuvieron diferencias significativas con los no co-infectados respecto a: demanda de $\mathrm{O}_{2}$ (p: 0,90 ), requerimiento de asistencia respiratoria mecánica ( $\mathrm{p}: 0,90)$ y duración de la estadía hospitalaria (p: 0,38). Tampoco se encontró asociación de las co-infecciones con los diagnósticos de egresos tales como neumonía (p: 0,20$)$ y bronquiolitis (p: 0,19).

\section{Discusión}

Desde su descubrimiento en el año 2005, HBoV ha sido detectado en muestras respiratorias y de materia fecal alrededor de todo el mundo. En el presente estudio se detectó ADN de bocavirus en 7\% (n: 78) de las muestras -en Tucumán en 5,9\% y en Santa Fe 8,5\%- hallazgo que concuerda con trabajos de otras regiones que reportan tasas entre 0,9 y $33 \%{ }^{7,13-25}$. En Argentina se ha estudiado la prevalencia de $\mathrm{HBoV}$ en la provincia de Córdoba, donde obtuvieron resultados entre 6,8 y $27,3 \%$ desde 2007 a $2011^{14,17}$; y en Buenos Aires, donde el virus se encontró en porcentajes que variaron entre 10,8 y 18,4\%, entre los años 2007 y $2010^{24,25}$. En lo que respecta al resto de América Latina, en Brasil trabajos reportan 2,44\% de prevalencia ${ }^{15}$; en Chile $21,8 \%{ }^{22}$; en Perú, $25,1 \%{ }^{24}$ y en Nicaragua, $33,3 \%{ }^{24}$. El amplio rango de frecuencia con que se detecta el genoma de $\mathrm{HBoV}$, podría deberse a las variaciones en la elección de la secuencia blanco y/o de la metodología. Las tasas más elevadas corresponden a Perú y Nicaragua, postulándose también, que la circulación del virus en estas áreas se encontraría favorecida por las condiciones ambientales ${ }^{24}$.

Se detectó $\mathrm{HBoV}$ en ambas provincias en todos los meses del año, a excepción del mes de noviembre. En general, los porcentajes mensuales variaron entre 2,1 y $12,0 \%$. No obstante, durante el mes de febrero se detectó $\mathrm{HBoV}$ en $37,5 \%$ de los pacientes, porcentaje condicionado por el elevado número de casos registrados en dicho mes en la provincia de Santa Fe (63,6\%). Los porcentajes de positividad en ambas provincias fueron ligeramente superiores en los meses correspondientes a las estaciones de primavera y verano. Si bien la mayoría de los autores reportó tasas superiores del virus en los meses de otoño e invierno ${ }^{13-15,17,22}$, estudios realizados en China ${ }^{7,19}$ muestran una mayor frecuencia de $\mathrm{HBoV}$ en los meses de primavera y verano. Zhou y cols., realizaron un estudio durante tres años y hallaron tasas más elevadas en los meses de verano ${ }^{19}$.

Con respecto a la distribución por grupos de edad, $92 \%$ de los casos fueron reportados en pacientes bajo 2 años de edad, detectándose bajo 6 meses de edad en $44 \%$, hallazgo 
que también fue observado en otras publicaciones ${ }^{6,10,13}$ lo que indica que $\mathrm{HBoV}$, al igual que otros virus respiratorios, tiende a infectar a niños bajo 2 años.

Del $41 \%$ de pacientes que presentaban antecedentes mórbidos, el principal fue la prematurez (22\%), factor que predispone a las IRA ${ }^{3}$, seguido de cardiopatía congénita $(13 \%)$ y desnutrición ( $9 \%)$. Se encontró relación estadísticamente significativa entre la presencia de antecedentes mórbidos y la aparición de enfermedad grave. Teniendo en cuenta que el principal antecedente fue la prematurez, esto sería coincidente con lo reportado para VRS, ante el cual los pacientes prematuros tienen mayor riesgo de desarrollar IRAs complicadas ${ }^{28}$.

Los síntomas más frecuentes fueron tos, disnea, fiebre, presencia de estertores subcrepitantes y tiraje. Rinorrea y sibilancias estuvieron presentes en 53\% de los pacientes. Estos síntomas no muestran diferencias a los que normalmente se manifiestan en las IRAs debidas a otros virus ${ }^{29}$.

La bibliografía referida a $\mathrm{HBoV}$ reporta su asociación, tanto a IRAs altas (tos, fiebre, conjuntivitis, faringitis, laringitis y otitis) como a IRAS bajas (neumonía, bronquitis, bronquiolitis, obstrucción bronquial y síndrome coqueluchoide) $)^{30}$. En nuestro estudio, los diagnósticos de egreso más frecuentes fueron neumonía, bronquitis y bronquiolitis, coincidiendo con resultados de un estudio en Córdoba, donde encontraron que 79\% de los pacientes positivos para $\mathrm{HBoV}$ presentaban IRA baja como diagnóstico ${ }^{23}$. Lo mismo ocurre en trabajos publicados de China ${ }^{7,11}$, Brasil ${ }^{10}$ y Tailandia, que hallaron $\mathrm{HBoV}$ en aspirados nasofaríngeos de pacientes con neumonía ${ }^{31}$. Los episodios de sibilancia también fueron un diagnostico frecuente en nuestro estudio (15,6\%), hecho ya reportado, al igual que la asociación de $\mathrm{HBoV}$ con los episodios de exacerbación del asma ${ }^{13,32}$.

La frecuencia de co-detecciones con otros virus respiratorios fue elevada: $28,2 \%$. El más frecuente fue VRS seguido de parainfluenza 3; también se detectaron ADV, MPVh e influenza A. El número de co-detecciones hallado en nuestro trabajo, si bien es alto, es inferior al reportado por otros autores, cuyos porcentajes oscilaron entre 30 y $76 \%{ }^{7,13,14,19,22}$. Por este motivo, se debate si el virus causa infección respiratoria o es sólo un espectador de otras infecciones. No se encontró relación entre las coinfecciones y la aparición de enfermedad grave, al igual que en otras publicaciones ${ }^{7,10,19}$, en las que se describió que no existían diferencias significativas en la expresión clínica de los pacientes según estuvieran co-infectados o $\mathrm{HBoV}$ fuera el único agente detectado, en lo que respecta a signos y síntomas ni en el desarrollo de enfermedad grave.
Nuestro estudio tiene algunas limitaciones: No se realizó la detección de $\mathrm{HBoV}$ en un grupo control debido a que uno de los mayores problemas en estudios de casos-control radica en la selección de un grupo control adecuado, ya que debe valorarse que este grupo no haya padecido un cuadro de vías respiratorias con anterioridad, al menos 5 o 6 semanas previas. Otra limitación del trabajo reside en que el número de co-detecciones puede haber sido subestimado ya que para la detección de los virus respiratorios clásicos se utilizó una técnica de menor sensibilidad como es la inmunofluorescencia.

Para concluir, hemos demostrado que $\mathrm{HBoV}$ circula en Santa Fe y Tucumán y lo detectamos en pacientes con IRA, principalmente niños bajo 2 años de edad. Futuros estudios son necesarios para diferenciar una infección reciente.

Agradecimientos. A M. Pilar Adamo del Instituto de Virología "J. M. Vanella", Facultad de Medicina de la Universidad Nacional de Córdoba, quien gentilmente nos cedió el control positivo de bocavirus.

\section{Resumen}

Introducción: Un alto porcentaje de las infecciones respiratorias agudas (IRA) permanece sin diagnostico etiológico, por lo cual se investigan nuevos patógenos continuamente. Bocavirus humano ( $\mathrm{HBoV}$ ) fue descubierto en 2005, como un nuevo miembro de la familia Parvoviridae y propuesto como causante de IRA. Objetivos: Investigar la prevalencia de $\mathrm{HBoV}$ en niños bajo 5 años de edad, hospitalizados por IRA en dos provincias de Argentina: Santa Fe y Tucumán y describir aspectos epidemiológicos y clínicos asociados a su detección. $M a-$ teriales y Métodos: Se estudiaron retrospectivamente los aspirados nasofaríngeos (ANF) de pacientes bajo 5 años de edad, con diagnóstico de IRA, hospitalizados durante el año 2013. La presencia de HBoV se detectó mediante la RPC de punto final descripta por Allander y cols. Los virus tradicionales se estudiaron mediante inmunofluorescencia. Datos personales, clínicos y epidemiológicos se recolectaron en una planilla estandarizada. Resultados: $\mathrm{HBoV}$ fue detectado en $7 \%$ de las muestras con prevalencia en primavera y verano; y principalmente en pacientes bajo 2 años de edad. Se registró co-detecciones en $22 \%$ de los casos. Discusión: Hemos detectado HBoV en estas dos provincias de Argentina; estudios posteriores deberán efectuarse para determinar si se trata de una infección reciente o una excreción prolongada del virus. 


\section{Referencias bibliográficas}

1.- Ministerio de Salud de la Nación Argentina. Recomendaciones para la vigilancia, prevención $\mathrm{y}$ atención de las infecciones respiratorias agudas en Argentina. Actualización mayo de 2015. www.msal.gov.ar

2.- Hasegawa K, Camargo C A. Air way microbiota and acute respiratory infection in children. Expert Rev Clin Immunol 2015; 11 (7): 789-92.

3.- Etiler N, Velipasaoglu S, Aktekin M. Incidence of acute respiratory infections and the relationship with some factors in infancy in Antalya, Turkey. Pediatr Int 2002; 44 (1): 64-9.

4.- Berry M, Gamieldien J, Fielding B C. Identification of new respiratory viruses in the new millennium. Viruses 2015, 7: 996-1019.

5.- John C, Kumund K, Stephen A, Mark H. Undiagnosed respiratory viruses in children. Pediatrics 2008; 121: e631-37.

6.- Allander T, Tammi M, Eriksson M, Bjerkner A, Tiveljung-Lindell A, Andersson B. Cloning of a human parvovirus by molecular screening of respiratory tract samples. Proc Natl Acad Sci USA 2005; 102: 12891-6.

7.- Xu L, He X, Zhang D M, Feng F S, Wang Z, Guan L L, et al. Surveillance and genome analysis of human bocavirus in patients with respiratory infection in Guangzhou, China. PLoS One 2012; 7 (9): e44876.

8.- Zhao B, Yu X, Wang C, Teng Z, Wang C, Shen J, et al. High human bocavirus viral load is associated with disease severity in children under five years of age. PLoS One 2013; 8 (4): e62318.

9.- Babkin I V, Tyumentsev A I, Tikunov A Y, Zhirakovskaia E V, Netesov S V, Tikunova N V. A study of the human bocavirus replicative genome structures. Virus Res 2015; 195: 196-202.

10.- Proença-Modena J L, Gagliardi T B, Paula F E, Iwamoto M A, Criado M F, Camara A A, et al. Detection of human bocavirus mRNA in respiratory secretions correlates with high viral load and concurrent diarrhea. PLoS One 2011; 6 (6): e21083.

11.- Zhao M, Zhu R, Qian Y, Deng J, Wang F, Sun Y, et al. Prevalence analysis of different human bocavirus genotypes in pediatric patients revealed intra-genotype recombination. Infect Genet Evol 2014; 27: 382-8.

12.- Pogka V, Moutousi A, Kossyvakis A, Kalliaropoulos A, Sgouras D N, Giannaki M, et al. Genetic variability of human metapneumo and bocaviruses in children with respiratory tract infections. Influenza Other Respir Viruses 2014; 8 (1): 107-15.

13.- García-García M L, Calvo Rey C, Pozo Sánchez F, Vázquez Álvarez M C, González Vergaz A, Pérez-Breña P, et al. Human bocavirus infections in Spanish 0-14 year-old: clinical and epidemiological characteristics of an emerging respiratory virus. An Pediatr (Barc) 2007; 67 (3): 212-9.

14.- Moreno L, Eguizábal L, Ghietto L M, Bujedo E, Adamo M P. Human bocavirus respiratory infection in infants in Córdoba, Argentina. Arch Argent Pediatr 2014; 112 (1): 70-4.

15.- Caccia E R, Watanabe A S, Carraro E, Leal E, Granato C, Bellei N. Frequency of human bocavirus respiratory infections among at-risk patients in São Paulo, Brazil. Rev Inst Med Trop Sao Paulo 2012; 54 (6): 307-10.

16.- Bastien N, Brandt K, Dust K, Ward D, Li Y. Human bocavirus infection, Canada. Emerg Infect Dis 2006; 12 (5): 848-50.

17.- Ghietto L M, Cámara A, Zhou Y, Pedranti M, Ferreyra S, Frey T, et al. High prevalence of human bocavirus 1 in infants with lower acute respiratory tract disease in Argentina, 2007. 2009. Braz J Infect Dis 2012; 16 (1): 38-44.

18.- Kleines M, Scheithauer S, Rackowitz A, Ritter K, Häusler M. High prevalence of human bocavirus detected in young children with severe acute lower respiratory tract disease by use of a standard PCR protocol and a novel real-time PCR protocol. J Clin Microbiol 2007; 45 (3): 1032-4

19.- Zhou L, Zheng S, Xiao Q, Ren L, Xie X, Luo J, et al. Single detection of human bocavirus 1 with a high viral load in severe respiratory tract infections in previously healthy children. BMC Infect Dis 2014; 14: 424.

20.- Principi N, Piralla A, Zampiero A, Bianchini S, Umbrello G, Scala A, et al. Bocavirus infection in otherwise healthy children with respiratory disease. PLoS One 2015; 10 (8): e0135640.

21.- Wu J J, Jin Y, Lin N, Xie Z P, Yu J M, Li J S, et al. Detection of human bocavirus in children with acute respiratory tract infections in Lanzhou and Nanjing, China. Biomed Environ Sci 2014; 27 (11): 841-8.

22.- Flores C J C, Vizcaya A C, Araos B R, Montecinos P L, Godoy M P, ValienteEcheverría F, et al. Human bocavirus in Chile: clinical characteristics and epidemiological profile in children with acute respiratory tract infections. Rev Chilena Infectol 2011; 28 (6): 504-11.

23.- Ghietto L M, Majul D, Ferreyra Soaje P, Baumeister E, Avaro M, Insfrán C, et al. Comorbidity and high viral load linked to clinical presentation of respiratory human bocavirus infection. Arch Virol 2015; 160 (1): 117-27.

24.- Salmón-Mulanovich G, Sovero M, Laguna-Torres V A, Kochel T J, Lescano A G, Chauca $\mathrm{G}$, et al. Frequency of human bocavirus $(\mathrm{HBoV})$ infection among children with febrile respiratory symptoms in Argentina, Nicaragua and Peru. Influenza Other Respir Viruses 2011; 5 (1): $1-5$.

25.- Marcone D N, Carballal G, Ricarte C, Echavarría M. Respiratory viral diagnosis by using an automated system of multiplex PCR (FilmArray) compared to conventional methods. Rev Argent Microbiol 2015; 47 (1): 29-35.

26.- Martin E T, Kuypers J, McRoberts J P, Englund J A, Zerr D M. Human bocavirus 1 primary infection and shedding in infants. J Infect Dis 2015; 212 (4): 516-24

27.- Centers for Disease Control and Prevention (CDC). CDC real time RTPCR (rRTPCR) protocol for detection and characterization of influenza A (H1N1). Available at http://www. who.int/csr/resources/publications/swineflu/ CDCrealtimeRTPCRprotocol_20090428.pdf

28.- Liese J G, Grill E, Fischer B, RoecklWiedmann I, Carr D, Belohradsky B H. Incidence and risk factors of respiratory syncytial virus-related hospitalizations in premature infants in Germany. Eur J Pediatr 2003; 162 (4): 230-6.

29.- Schildgen O, Müller A, Allander T, Mackay I M, Völz S, Kupfer B, et al. Human bocavirus: passenger or pathogen in acute respiratory tract infections? Clin Microbiol Rev 2008; 21 (2): 291-304.

30.- Moreno C, Solís Y, O’Ryan M. Human bocavirus: studies in the literature and in Chile. Rev Chilena Infectol 2009; 26 (6): 504-10.

31.- Fry A M, Lu X, Chittaganpitch M, Peret T, Fischer J, Dowell S F, et al. Human bocavirus: a novel parvovirus epidemiologically associated with pneumonia requiring hospitalization in Thailand. J Infect Dis 2007; 195 (7): 1038-45.

32.- Allander T, Jartti T, Gupta S, Niesters HG, Lehtinen P, Osterback R, et al. Human bocavirus and acute wheezing in children. Clin Infect Dis 2007; 44 (7): 904-10. 